\title{
The Psychological Effect of Visual and Auditory Stimuli on the Road Traffic Noise Perception
}

\author{
Gil-Soo JANG ${ }^{1)}$, Hoon SHIN ${ }^{2)}$, Min-Jeong SoNG ${ }^{3)}$ and Chan $\mathrm{KoOK}^{4)}$ \\ ${ }^{1)}$ Department of Architectural Engineering, Dongshin University \\ ${ }^{2)}$ Department of Architectural Engineering, Graduate School Chonnam National University \\ ${ }^{3)}$ Bio-Housing Research Institute, Chonnam National University \\ ${ }^{4)}$ Department of Landscape Architecture, Dongshin University, \\ 252 Daeho-Dong, Naju, Jeonnam 520-714, Korea \\ E-mail: gsjang@dsu.ac.kr
}

(received on October 11, 2007, accepted on January 9, 2008)

\begin{abstract}
Several studies on audio-visual interactions have shown that the visual stimulus can influence loudness of traffic noise. And it was shown that the auditory stimulus such as music in the street can reduce the road traffic noise perception. On the base of these results, a series of experimental studies were carried out and reviewed the psychological effect of visual and auditory stimuli on the perception of road traffic noise.

The first experiment was designed to assess the visual effect of twelve roadsides with different landscapes on the road traffic noise perception. As a result, approx. 3 to $5 \mathrm{~dB}(\mathrm{~A})$ of psychological reduction was seen in places where natural landscape was preferred, compared to the others, although the noise levels were similar. The second experiment was designed to evaluate the effect of visual screening from adjacent roads on road traffic noise perception by means of ME method in a laboratory. As the result, the effect of psychological reduction was observed at $65 \mathrm{~dB}(\mathrm{~A})$ or lower. Especially, the barrier which screening optically from adjacent roads led to 5 to $10 \%$ of loudness reduction effect, compared to non-screening cases.

Finally, the third experiment was designed to evaluate the effect of visual and auditory stimuli with ME method and 7-point SD rating scale in a laboratory. And it was found that up to $10 \%$ of loudness reduction and about $2 \mathrm{~dB}(\mathrm{~A})$ of noise perceptional reduction were seen at $65 \mathrm{~dB}(\mathrm{~A})$ or lower.
\end{abstract}

Key words: road traffic noise, visual and auditory stimulus, psychological loudness reduction, barrier type

\section{Introduction}

As one of the important emotion-evoking stimuli in relation to our five sensations, sound is used as an essential means for human communications. However, loud sounds generated in a city often cause psychological stress or annoyance. Because of such negative influences of sound, various attempts have been done including reducing the acoustic power of noise sources, installing soundproof walls, developing sound insulating/absorbing materials, and designating noise control districts to reduce the level of noise. Some of these attempts were substantially effective; however, almost all of them are considered the physical control of noises only.

On the contrary, there are unconventional attempts to analyze and evaluate urban environmental noises in their various contexts as well as in the auditory aspect. Because urban environmental sounds are perceived not simply through hearing sensation, but through a combination of multi-sensations. In other words, the conventional approaches neglected the fact that the human senses interact rather than function merely independent of each other. Specifically, human multi-sensory interaction among vision, hearing, smell, touch and taste should be considered in evaluating environmental sounds.

In general, noise is defined not as loud or powerful sound, but unwanted or undesirable sound. It suggests that not only acoustic factors but also nonacoustic factors act on noise perception. In this view point, some previous studies attempted to address the problem of noises in the aspect of the human multisensory interaction, instead of simply in the aspect of hearing. Of them, the most attempts relate to interaction between sound and visual stimuli. The typical studies include the followings. Abe et al. (1999) argued that the presentation of white noise in combination with a waterfall scene led to decreasing loudness 
Table 1. Nine attributes of semantic differential scales for assessment of environmental sound quality.

\begin{tabular}{lll|ll}
\hline \multicolumn{3}{c|}{ Attributes of } & scales & \multicolumn{2}{c}{ Korean terms } \\
\hline 1. Quite & - & 7. Loud & 1.작은 - & 7.큰 \\
1. Pleasant & - & 7. Unpleasant & 1.유쾌한 - & 7.짜증나는 \\
1. Little attending & - & 7. Very attending & 1.빈약한 - & 7.곽 차는 \\
1. Organized & - & 7. Disorganized & 1.정돈된 - & 7.산만한 \\
1. Nearby & - & 7. Far & 1.가까운 - & 7.먼 \\
1. Steady & - & 7. Unsteady & 1.연속적인 - & 7.불연속적인 \\
1. Established & - & 7. Evolutive & 1.고정된 - & 7.변화하는 \\
1. Hubbub & - & 7.Distinct & 1.멍멍한 - & 7.또렷한 \\
1. Monotonous & - & 7.Varied & 1.단조로운 - & 7.다양한 \\
\hline
\end{tabular}

of the represented noise. Viollon et al. (1999) demonstrated that the results of evaluating the singing of birds were considerably different when adding acoustic stimuli to urban landscape vs. forest landscape. In addition, Hashimoto and Hatano (2001) suggested that image presentation could mitigate the annoyance of vehicle noises, which is quite powerful to the extent that increasing the noise level by more than $10 \mathrm{~dB}$ was required to bring about the equivalent reaction in the absence of image. Kuwano et al. (2001) reported that audio-visual interaction affected not only the volume of sound but aesthetic evaluations of listeners and at this moment, the green leaves of trees played an important role. And Iwamiya et al. (2000) put forth that adding music has influence upon the impression of a given landscape or space, and that accounts for such the positive synergy of landscape and music as resonance.

The findings mentioned above suggest the significance of psychological effect on noise perception, and further the potential for psychological control as well as physical control of noise. This study aims at identifying the effect of visual and auditory stimuli on the road traffic noise perception through a series of psychoacoustic experiments.

\section{Visual effect of urban spaces on noise percep- tion}

This study was attempted to quantify the visual effect on noise perception through field experiments. For the purpose, different urban spaces with combined acoustic and eco-friendly factors were extracted, and the subjective responses were surveyed. For its methodology, 12 sites with different spatial characteristics were selected, and subjects were asked to evaluate the acoustic environments of the given spaces by seven step SD scale. Here, urban spatial characteristics mean the level of noise, visual features and spatial occupation, and specifically indicate characteristics caused by the presence of waterside space, the occupation degree of forest, the ratio of resting spaces, the degree of urban vitality and others. Total
44 university students (aged 23.2 on the average) participated in this field survey. Existing field surveys have employed mainly passerby interview or questionnaire by surveyors; however, this study adopted the method in which same subjects moved to twelve sites and stayed for a certain period of time for assessment. This was intended to minimize the misunderstanding about rating vocabulary, which may be caused in evaluation by unspecified people, and to ensure consistent evaluations among subjects. In the twelve sites, total 528 questionnaire sheets were collected and of them, 522 sheets excluding non-responded were selected as valid ones. Questionnaire consisted of assessment of total amenity of the site (1. not comfortable-7. very comfortable), assessment of satisfaction with 5 environmental elements (thermal, vision, air quality, lighting, smell) with sound (1. worst-7. best), assessment of disturbance degree of conversation (1. worst-7. best), and assessment of sound quality of 9 attributes by the seven step satisfaction. Table 1 shows the items for assessment of environmental sound quality.

According to the findings, total amenity (TA) had significant correlation with individual environmental factors, especially landscape, air quality and offensive smell. In other words, it is considered that the evaluation on the TA we usually perceive depends not only on acoustic environment but also significantly on visual and olfactory factors including landscape or air quality. Although it is known that the perception of acoustic environment typically has direct relation to physical noise level or traffic volume, it also shows significant correlation with their perceived levels, so that it could be concluded that ambient environmental factors have indirect influence upon the results of evaluation.

After simplification of the correlation between TA and five environmental factors through subjective TA evaluation, assuming it as primary linear model and performing regression analysis, the following formula was derived. 
Table 2. Correlation coefficient between TA and environmental elements.

\begin{tabular}{|c|c|c|c|c|c|c|c|}
\hline & TA & acoustic & thermal & landscape & air & lighting & smell \\
\hline $\mathrm{TA}$ & $1.00 *$ & & & & & & \\
\hline acoustic & $0.48^{*}$ & 1.00 & & & & & \\
\hline thermal & $0.41^{*}$ & $0.43 *$ & 1.00 & & & & \\
\hline landscape & $0.57^{*}$ & $0.35^{*}$ & $0.30^{*}$ & 1.00 & & & \\
\hline air & $0.59 *$ & $0.50^{*}$ & $0.34^{*}$ & $0.55^{*}$ & 1.00 & & \\
\hline lighting & $0.39^{*}$ & $0.26^{*}$ & $0.47^{*}$ & $0.37 *$ & $0.41 *$ & 1.00 & \\
\hline smell & $0.53 *$ & $0.35^{*}$ & $0.31 *$ & $0.47 *$ & $0.57^{*}$ & $0.38^{*}$ & 1.00 \\
\hline
\end{tabular}

TA: Total Amenity *: $p<0.01$

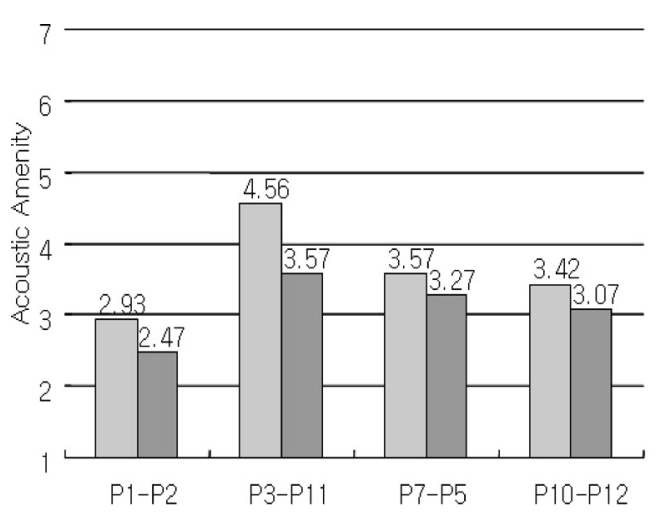

(a) Mean value of acoustic amenity

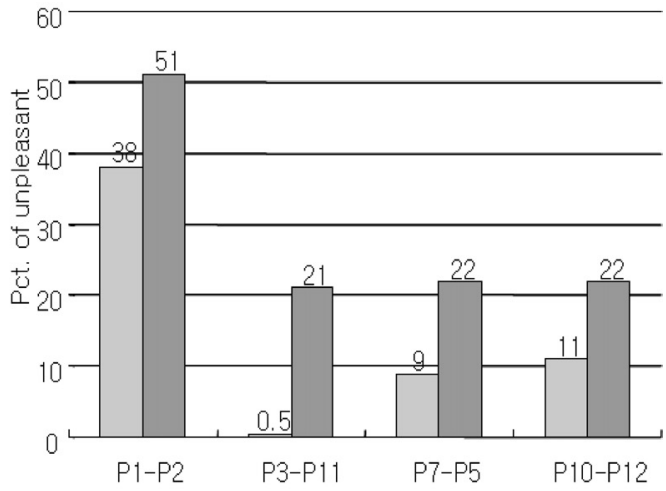

(b) Percentage of unpleasantness response

Fig. 1. Comparative difference of acoustic amenity according to the satisfaction of landscape.

$$
\begin{aligned}
\mathrm{TA}= & 0.162 *[\text { Acoustic }]+0.121 *[\text { Thermal }] \\
& +0.266 *[\text { Landscape }]+0.219 *[\text { Air }] \\
& +0.047[\text { Lighting }]+0.178 *[\text { Smell }]+0.199
\end{aligned}
$$

Except for the evaluation item of lighting environment, all variables had significant correlation with the whole environment and among them, landscape and air environment showed highest contribution, while acoustic environment showed relatively low contribution. It could be deduced that surrounding environmental elements indirectly influence it as significant correlation is found by the degree of recognition of such elements.

Out of twelve survey sites, responses of acoustic amenity and unpleasantness were analyzed between some sites (P1, P3, P7, and P10) of preferred landscape with the other sites (P2, P11, P5, P12) of negative landscape where physical noise levels and traffic situations were similar but landscape satisfaction was generally high or low by the view point of its abundant natural factors. The comparison of mean values by the degree of acoustic amenity i.e., satisfaction with sound element (1. worst-7. best) to the place is shown in (a) of Figure 1 . And the comparison of percentage ( $\%$ of subjects who responded as 6 and 7 in seven scale) corresponding to the degree of discomfort or temper as opposed to that of comfort is shown in (b). As shown in Figure 1, the higher the satisfac- tion with landscape was, the higher became the acoustic amenity in survey sites. Also, a consistent phenomenon was shown in the result of comparison on the percentage of the degree of discomfort. Specially, difference in the noise perception between P3 and P11 (P3-P11) of survey sites was most, which was equivalent to 1 out of 7 . On the average, the differences ranged from 0.3 to 0.5 . This phenomenon was regarded as a generally consistent pattern, because the compared spaces included downtown road, park, pedestrian street and square. In other words, visual stimulus seems to have direct relationship with noise perception. Then, if any difference in subjective amenity perception is converted into physical noise, how much will visual stimulus contribute to the result? As 1 out of 7 scale showed about $11 \mathrm{~dB}(\mathrm{~A})$ of difference from the relationship between physical noise level and subjective amenity, 0.3 to 0.5 of perception differences were equivalent to 3.3 to 5.5 $\mathrm{dB}(\mathrm{A})$, if converting the difference in visual information perception into physical noise level. Thus, the authors regarded that as psychological effect of environmental noise perception due to difference in visual stimuli.

\section{Optical screening effects of barriers}

The purpose of this experiment is to quantify the 


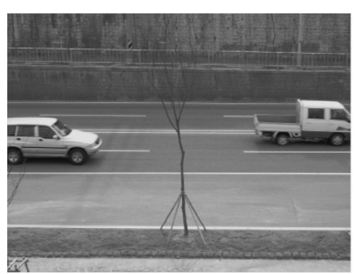

a) B1

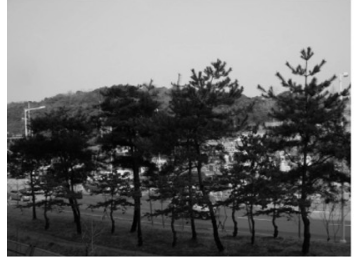

c) B3

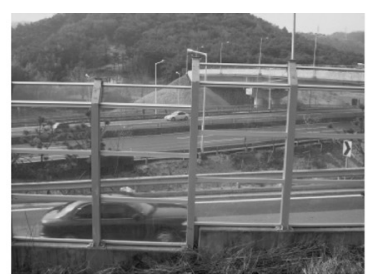

b) B2

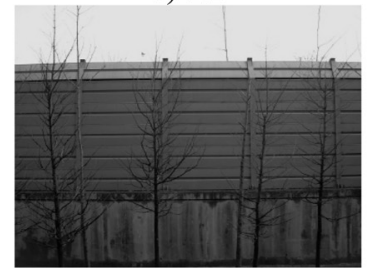

d) B4

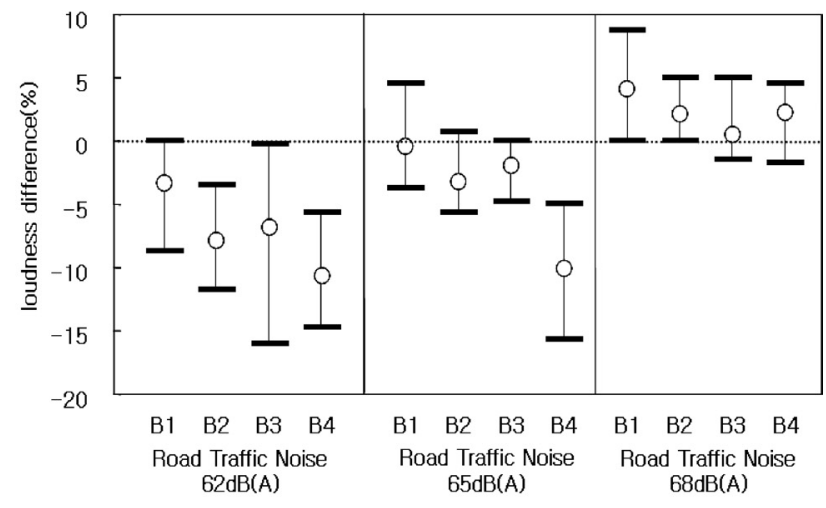

* Two dash lines mean the upper and lower quartiles of loudness. Circular sign means the average of loudness.

Fig. 2. The effect of the noise barrier image on the loudness of noise at the same noise level.

effect of visual and auditory information on the loudness of noise. For the purpose, a psycho-acoustic experiment was performed in the manner of having subjects listen to precompiled sounds via headphones, and then evaluate their own responses by means of Magnitude Estimation (ME) method. This method was designed to offer the reference sound only prior to the presentation of various visual stimuli, and subsequently the relative magnitude of noise after the presentation of the visual stimuli, which enables to quantify the effect of visual stimuli. Used as the target noises in this experiment, the road traffic noises were characterized by relatively in their levels, and were compiled after field recording. The target level was set to $65 \mathrm{~dB}(\mathrm{~A})$, and the levels of road traffic noises $62 \mathrm{~dB}(\mathrm{~A}), 65 \mathrm{~dB}(\mathrm{~A})$ and $68 \mathrm{~dB}(\mathrm{~A})$ prepared with taking $\pm 3 \mathrm{~dB}(\mathrm{~A})$ as their relative level.

Visual stimuli presented to subjects were the visual information showing the degree of shielding against the road depending on the presence of noise barrier.

The subjects participated in this experiment consisted of male and female university students with normal hearing (male: 7/female: 7) in their twenties. Prior to the main experiment, the subjects became fully familiar with experiment methods and descriptions of presentation, and especially were asked to evaluate the images after watching them to the full in the light of evaluating the effect of visual stimuli. However, the purpose of the experiment was not mentioned at all. For ME method employed as the evaluation tool used in this experiment, the loudness of given stimulus should be compared with that of a reference stimulus. Thus, road traffic noises were presented as reference stimulus which was regarded as the value 100, and the comparison stimuli presented successively were evaluated in respect to the relative loudness.

Figure 2 shows the responses of fourteen subjects converted into the average and quartiles, and diagrams the relative differences in the loudness of noise on the basis of reference stimulus without any visual stimulus. Figure 2 brings us to two observations as follows: one is that for $65 \mathrm{~dB}(\mathrm{~A})$ or lower noises, their relative loudness decreased when combined with visual information, compared to those without visual information; however, if the noise exceeds $65 \mathrm{~dB}(\mathrm{~A})$, the loudness increased. In other words, non-acoustic factors, especially visual stimuli have positive influence on the loudness of noise below a certain tolerance limit. As the other observation, it was found that the presentation of visual information affected as much as about $5-10 \%$ the loudness of noise regarding noise barrier. In the case of noise barrier where no running vehicle is seen, the relative loudness was the lowest, which suggests physical soundproof effect as well as psychological effect.

\section{Psychological effect of visual and auditory in- formation}

In general, environmental noises are recognized by the five senses simultaneously-not simply with visual information. So, in addition to the psychological effect of visual stimulus described previously, the authors attempted to clarify the additional effect of auditory information and to evaluate its psychological effect of the road traffic noise by rating the discomfort of noise with 7-point rating scale. During the laboratory experiment, road traffic noises of $\mathrm{L}_{\mathrm{Aeq}} 55 \mathrm{~dB}$, $60 \mathrm{~dB}, 65 \mathrm{~dB}, 70 \mathrm{~dB}$, and $75 \mathrm{~dB}$ were presented to the subjects via a headphone. Also the auditory stimuli of signal music and ambient music were added to the traffic noise as a masker or a background sound. At this moment, the levels of auditory information were lowered as much as $-5 \mathrm{~dB}$ at $\mathrm{L}_{\text {Aeq }} 55 \mathrm{~dB}$ to $65 \mathrm{~dB}$ of road traffic noise levels, and $-10 \mathrm{~dB}$ at $\mathrm{L}_{\mathrm{Aeq}} 70 \mathrm{~dB}$ above, which is based on the level considered the 


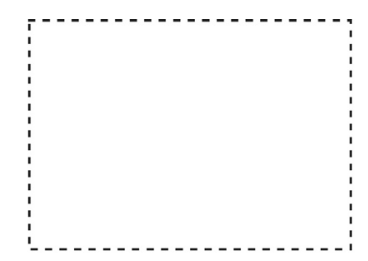

a) $\mathrm{B}$ - no image

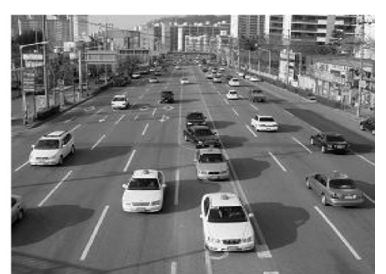

b) V1 - road

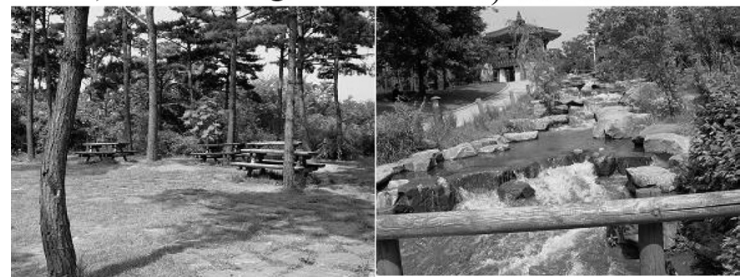

c) V2 - green area

d) V3 - green \& water

Fig. 3. The type of visual information.

most suitable for added sound in the preliminary test. Visual information projected onto the screen was three kinds of scenes as shown in Figure 3. And auditory stimuli were signal music (S1) and green music (S2). At this time, a case without visual information presented was included as a reference scene.

All experiments were carried out with 43 normalhearing subjects trained in preliminary test (28 male students and 15 female students). Subjects were fully familiarized with the experiment methods to be followed and conditions to be given, and especially asked to evaluate after looking fully at the visual images presented during a given time; however, not informed the purpose of experiment at all, to avoid any bias concerning the experiment. The presented stimuli which were composed in a random order were evaluated by means of ME method. For ME method, the subjects listened to a fixed reference stimulus set to 100 and subsequently comparative stimuli presented, and were asked to evaluated their relative loudness. In addition, for noise discomfort, each of the noises presented was rated on the basis of 7-point SD scale (1: not at all-7: very).

Figure 4 shows the length of time for and the sequence in which comparative stimuli were presented in this experiment. In Figure 4, ' $\mathrm{V}$ ' and ' $\mathrm{N}$ ' means the visual information and the road traffic noise, also ' $\mathrm{S}$ ' means the auditory stimulus. After listening noise as reference stimuli and auditory information as comparative stimuli for 43 seconds, subjects were asked to evaluate the relative loudness of comparative stimuli within 5 seconds.

Figure 5 is the results of experiment by SD rating method. As a result of making a comparison between cases without and with visual information (V1, V2, $\mathrm{V} 3$ ), it was revealed that approx. $7 \mathrm{~dB}$ was reduced in those with visual stimuli. And the effect of auditory information (S1, S2) alone was equivalent to approx.

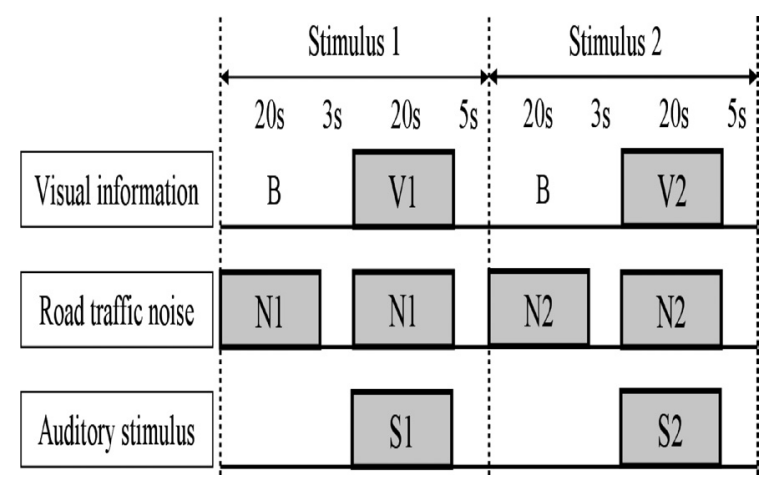

Fig. 4. Presentation sequence of stimuli.

$5 \mathrm{~dB}$. Although it is expected that there will be some difference in the effects depending on the characteristics of auditory information, the effects seem to work best at $65 \mathrm{~dB}(\mathrm{~A})$ or lower.

Figure 6 shows the results regarding the combined effects of visual and auditory stimuli. As seen in figure, the combined effects of visual and auditory stimuli tended to improve somewhat the noise perception levels, compared with that of respective stimulus. For example, if based on noise perception level 4 , the perceived noise level corresponding to each visual and auditory stimulus equals $59.8 \mathrm{~dB}(\mathrm{~A})$ from the regression equation of dash line; however, for the combined effect of visual and auditory stimuli, the noise perception noise level equals $62 \mathrm{~dB}(\mathrm{~A})$-about $2 \mathrm{~dB}$ of difference can be regarded as synergy of interacting visual and auditory stimuli. In other words, if both visual and auditory stimuli provided simultaneously, it may affect the noise perception.

If the above-mentioned effects would be applied to typical situations, what psychological effects would be expected? In the case of simply adding auditory stimuli (sign: $\mathrm{V} 1+(\mathrm{S} 1+\mathrm{S} 2)$ ), the psychological noise perception was not so significant. On the other hand, in the case of presenting eco-friendly visual stimuli (sign: V2 and V3) with abundant natural elements together with auditory stimuli (sign: S1, S2), perceived noise level reduced by approx. $2 \mathrm{~dB}$ at $65 \mathrm{~dB}$ below, while the level increased at $65 \mathrm{~dB}$ above. In conclusion, if visual and auditory information with higher amenity is introduced into urban spaces, the perceived acoustic environment can be improved and further at $65 \mathrm{~dB}$ below, even psychological discomfort can be considerably reduced.

The following two tendencies are observed in this experiment. One is that presenting visual and auditory stimuli at $65 \mathrm{~dB}(\mathrm{~A})$ below of noise levels caused the noise level to lower compared to that before presenting visual information, whereas the perceived noise levels to higher at $65 \mathrm{~dB}(\mathrm{~A})$ above. This finding suggests that visual or auditory stimulus have posi- 

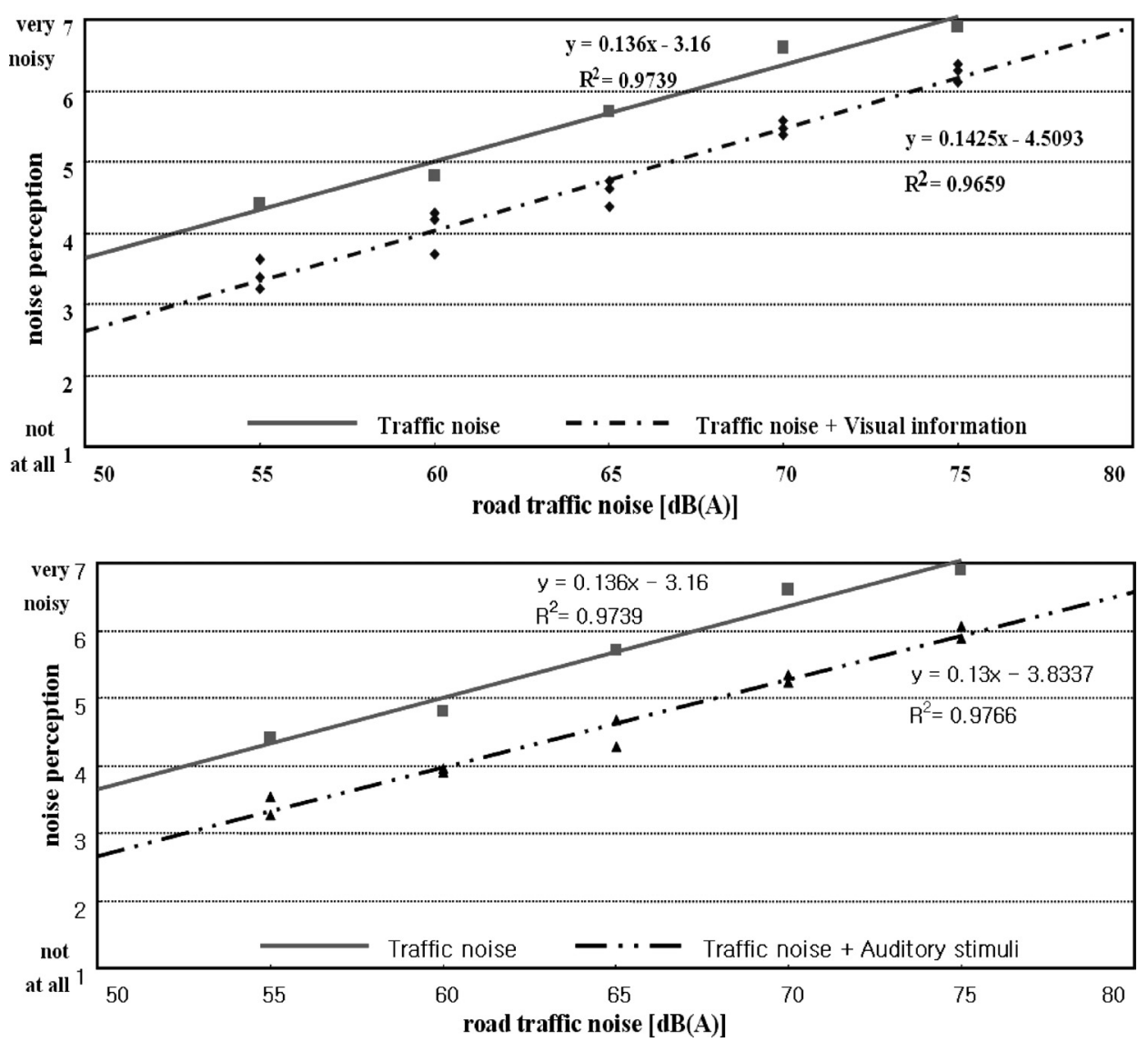

Fig. 5. The effect of the visual and auditory stimuli on the road traffic noise perception.

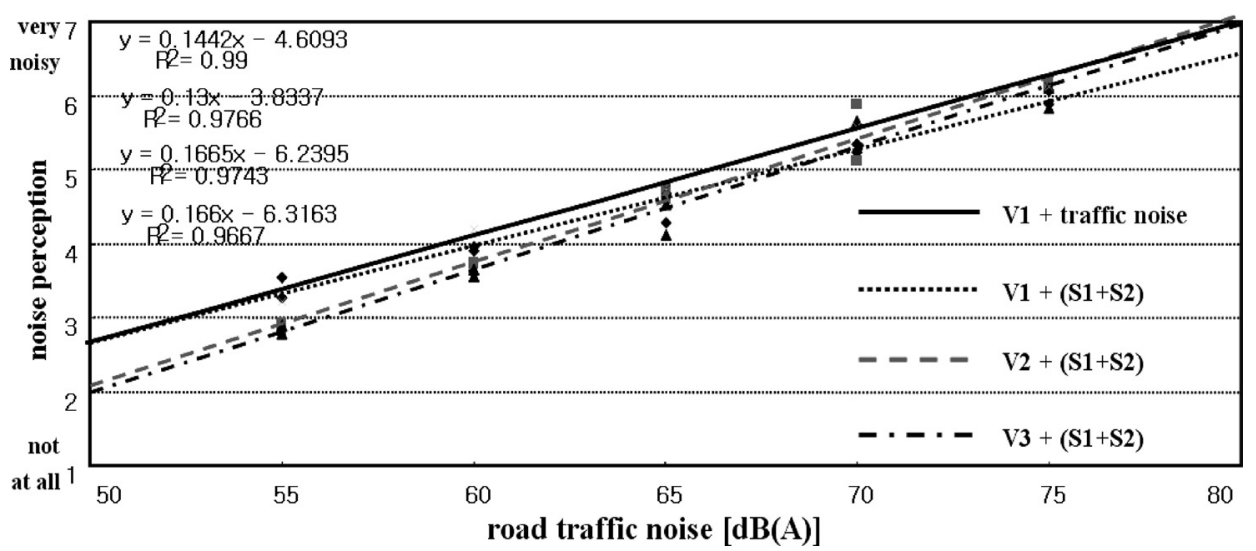

* Sign V1+(S1+S2) means the case of presenting visual stimuli (sign: V1) with auditory stimuli (sign: S1, S2) simultaneously.

Fig. 6. The combined effect of the visual and auditory stimuli on the road traffic noise perception.

tive influence upon the perceived noise level at a certain reference level considered as allowable noise. And, it indicates that in the psychological aspect, visual and auditory information affects the perceived noise level as much as up to $10 \%$ or so. It is also consistent with those obtained in the previous experiment and supports that visual and auditory information plays an important role in evaluating environmental noises.

\section{Results and discussion}

Assuming that visual and auditory stimuli affect the perceived noise level or discomfort level, what would be the effect of psychological reduction with quantitative noise level? The answer to this question is the very ultimate goal of this study.

Although this topic has been addressed fragmentarily in many studies, the authors attempted to approach it through a series of studies in an integrated 
manner. For methodology, the authors performed psycho-acoustic experiments in the fields as well as in the laboratory, and based upon the results, tried to determine quantitatively the psychological effect.

First experiment was designed to identify the effect of visual stimuli on psychological reduction of noise level. For the purpose, noise environment was evaluated in the fields of twelve target sites with different scenic features. As the result, about 3 to $5 \mathrm{~dB}(\mathrm{~A})$ of psychological reduction effect was shown in the sites where the noise levels were similar but natural landscapes were preferred, compared to other sites.

Based on the results of field experiments, laboratory experiments designed to control different variable factors were conducted. The first experiment was intended to evaluate the effect of visual shielding from adjacent roads on the perceived level of road traffic noise by means of ME method. As a result of doing so, significant psychological mitigation effects were observed at $65 \mathrm{~dB}(\mathrm{~A})$ below. Especially, absolutely shielding from running vehicles on the road led to 5 or $10 \%$ loudness reduction effect, compared to the case of non-shielding.

The third experiment was designed to evaluate the effect of visual and auditory stimuli by means of ME method and 7-point SD rating scale in the laboratory. In result, up to $10 \%$ of loudness reduction was seen at $65 \mathrm{~dB}(\mathrm{~A})$ below, and about $2 \mathrm{~dB}(\mathrm{~A})$ of psychological discomfort was reduced.

Putting the above-mentioned results together, introducing pleasant sounds and improving urban landscapes can be a useful approach to reducing the psychological noise perception at $65 \mathrm{~dB}(\mathrm{~A})$ below. This conclusion supports the necessity to perform integrated researches with taking multi-sensory integration (Schultz-Forkamp 2003). Furthermore, it also maintains the arguments that conceptual approach of soundscape enables the integrated researches (Raimbault 2003) and that one should look into a city as a whole and understand its sensory context in order to evaluate the urban environmental sounds in multilateral aspects.

\section{Acknowledgements}

This work was supported by the Korea Research Foundation Grant funded by the Korean Government (MOEHRD); (The Regional Research Universities Program/Biohousing Research Institute). A part of this study was presented at the 2nd International
Symposium on Design of Artificial Environments, which was held at Fukuoka in 2007.

\section{References}

Abe, K., Ozawa, K., Suzuki, Y., Sone, T. (1999) The effects of visual information on the impression of environmental sounds. Inter-noise 99: 1177-1182.

Schultz-Fortkamp, Brigitte (2003) Soundscape and community annoyance in the context of environmental impactassessment. Proceedings of internoise 2003: 28152824.

Jang, G.-S., Kook, C., Kim, S.-W. (2006) Field survey on the effect of ecological amenity on the recognition of road traffic noise. Proceedings of internoise 2006. paper 353 (CD Rom)

Watts, G., Chinn, L., Godfrey, N., (2002) The effects of vegetation on the perception of traffic noise. Applied Acoustics 56: 39-56.

Hashimoto, T., Hatano, S. (2001) Effects of factors other than sound to the perception of sound quality. 17th ICA Rome. CD-ROM.

Fastl, H. (2004) Audio-visual interactions in loudness evaluation. ICA 2004. Vol. 2: 1161-1166.

José Luis Carles, Isabel López Barrio, José Vicente de Lucio (1999) Sound influence on landscape values. Landscape and Urban Planning 43: 191-200.

Kawai, K., Yano, T. (2003) Relation between the overall impression of the sound environment and types and loudness of environmental sounds. JSV 250(1): 41-46.

Raimbault, M., Lanvandier, C., Berengier, M. (2003) Ambient sound assessment of urban environments: field studies in two French cities. Applied Acoustics 64: 1241-1256.

Song, M.-J., Shin, H., Back, G.-J., Jang, G.-S. (2007) Auditory and visual information effect on the loudness of noise. SB07 Seoul. Vol. 1: 645-650.

Sasaki, M. (1993) The preference of the various sounds in environment and the discussion about the concept of the soundscape design. J. Acoust. Soc. Jpa. (E) 14.3: 189-195.

Kuwano, S., Namba, S., Komatsu, M., Kato T., Hayashi, Y. (2001) Auditory and visual interaction in the aesthetic evaluation of environment. Empirical Studies of the Arts. 19(2): 191-200.

Viollon, S., Lavandier, C., Drake, C. (2002) Influence of visual setting on sound ratings in an urban environment. Applied Acoustics 63: 493-511.

Viollon, S. et al. (1998) Development of an experimental procedure suitable to test audio-visual interactions in the complex urban sound environments, Euro-noise $\mathbf{9 8}$. Vol. 2: 1095-1100.

Yang, W., Kang, J. (2005) Acoustic comfort evaluation in urban open public spaces. Applied Acoustics 66: 211229. 